\title{
Kulturelles und kommunikatives Gedächtnis Überlegungen zum Selbstverständnis der Pastoraltheologie in einer Mediengesellschaft
}

\author{
von Erich Garhammer
}

Pastoraltheologie versteht sich heute zumeist als Theorie der Praxis. Sie ist also nicht selber Praxis, sondern der Theoriehorizont von Praxis. Diese Theorie ist handlungsstiftend, bewahrt aber auch vor Handlungsdruck. Pastoraltheologie untersucht die das pastorale Handeln bestimmenden Theoriekonstrukte, etwa die Dekadenztheorie, die davon ausgeht, daß sich alles zum Schlechteren wandelt, die Fortschrittstheorie, die den Fortschritt als dominierende Kategorie der Geschichte annimmt, die Sündenbocktheorie, die schon immer weiß, wer woran schuld ist; aber auch elaboriertere Theorien wie die Säkularisierungstheorie, die von einem Abnehmen religiöser Weltdeutung ausgeht, oder die Modernisierungstheorie, die einen Übergang von der Moderne in die reflexive Moderne konstatiert. 1

Pastoraltheologie will also mit der Komplexität von Situationen vertraut machen und angesichts dieser Komplexität zum Handeln ermuntern. Aus diesem weiten Feld pastoraltheologischer Theoriebildung will dieser Beitrag ein kleines Mosaiksteinchen herausgreifen, nämlich das Selbstverständnis der Pastoraltheologie in einer Mediengesellschaft.

Pastoraltheologie gilt seit ihren Anfängen als Einbruchstelle aufklärerischer Positionen in den Bereich der Theologie. Die mariatheresianische Studienreform und ihre josephinische Zuspitzung werden oft als Brecheisen des bis dahin innertheologischen Diskurses interpretiert. Diese Sicht ist freilich insofern verkürzend, als sie von einer Dichotomie von Kirche und gesellschaftlicher Öffentlichkeit ausgeht und die Interdependenz beider Größen nicht in den Blick bekommt. Der Aufklärungs- und Jansenismushistoriograph P. Hersche ${ }^{2}$ hat hinlänglich nachgewiesen, wie gerade hinter den aufklärerischen Reformpositionen eine genuine Theologie steckte, nämlich das Theorem des Jansenismus. Von hier aus führen bruchlose Linien zu den Positionen der Aufklärer. Nicht die Dichotomie von theologischem und gesellschaftlichem Dis-

Prof. Dr. E. Garhammer ist Ordinarius für Pastoraltheologie und Homiletik an der Theologischen Fakultät Paderborn.

1 Vgl. dazu U. Beck, Risikogesellschaft. Auf dem Weg in eine andere Moderne,

Frankfurt/M. 1986 sowie H. Chr. Stoodt, Formen kirchlicher Arbeit an der

Schwelle von der Ind ustrie- zur Risikogesellschaft, in: PTh 80 (1991) 116-132.

2 P. Hersche, Der Spätjansenismus in Österreich, Wien 1977. 
kurs ist in diesem Zusammenhang erhellend, sondern die Analyse der Interaktion von theologischen Positionen mit dem Kommunikationsprofil einer bestimmten Öfentlichkeit.

Es soll im folgenden bei aller methodischen und sachlichen Verkürzung der Vorschlag gemacht und begründet werden, daß vor allem das Fach der Pastoraltheologie sich um die Analyse dieser Interaktion mühen sollte. Dabei will ich einen Begriff zugrunde legen, den J. Assmann ${ }^{3} \mathrm{im}$ Gefolge von M. Halbwachs geprägt hat: kulturelles und kommunikatives Gedächtnis. Unter kulturellem Gedächtnis versteht er die eine Gesellschaft fundierenden Erinnerungsgehalte wie Feste und Riten. „Feste und Riten sorgen im Regelmaß ihrer Wiederkehr für die Vermittlung und Weitergabe des identitätssichernden Wissens. ${ }^{44}$ Davon hebt Assmann das kommunikative Gedächtnis ab, das den Diskurs von zwei bis drei Generationen umschreibt. "Das kulturelle Gedächtnis ist ein Organ außeralltäglicher Erinnerung. Der Hauptunterschied gegenüber dem kommunikativen Gedächtnis ist seine Geformtheit und Zeremonialität seiner Anlässe.”5

In der heutigen Mediengesellschaft hat sich der von Assmann für jeden Gesellschaftstyp konstatierte Hiatus zwischen kulturellem und kommunikativem Gedächtnis erheblich zugespitzt: der aktuelle Diskurs hat ein viel schnelleres Verfallsdatum. G. Steiner6 spricht in diesem Zusammenhang vom "ephemeren Reißwolf" der Medien. Dennoch gehen gerade von diesen ephemeren Themen der Medien erhebliche Wirkungen in der Öffentlichkeit aus. So sehe ich es als eine - neben den oben beschriebenen anderen Aufgaben - notwendige Bemühung von Pastoraltheologie heute an, zwischen kulturellem und kommunikativem Gedächtnis im Medienzeitalter zu vermitteln. Daraus ergeben sich zwei nicht unerhebliche Konsequenzen: Pastoraltheologie schützt die Theologie vor der von H.J. Höhn ${ }^{7}$ so genannten Gefahr der permanenten Selbstzitation, indem sie das Kommunikationsprofil der Gesellschaft aufgreift und die dort transportierten Themen analysiert. Pastoraltheologie hat für die Theologie die Funktion eines "synchronen Korrektivs".

Pastoraltheologie macht aber zugleich in der medial beeinflußten Öffentlichkeit deutlich, daß das Neueste so neu gar nicht ist, daß der letzte Schrei durchaus schon an einen vorletzten anknüpft. Pastoral-

3 J. Assmann, Das kulturelle Gedächtnis. Schrift, Erinnerung und politische Identität in frühen Hochkulturen, München 1992.

4 Ebd., 57.

5 Ebd., 58.

6 G. Steiner, Von realer Gegenwart. Hat unser Sprechen Inhalt?, MünchenWien 1990.

7 H. J. Höhn, Signale der Zeit - Spuren des Glaubens, in: LS 45 (1994) 1-5; ders. ( $\mathrm{Hg}$.), Theologie, die an der Zeit ist. Entwicklungen - Positionen Konsequenzen, Paderborn 1992 sowie ders., Gegen-Mythen. Religionsproduktive Tendenzen der Gegenwart, Freiburg-Basel-Wien 1994. 
theologie hat also der Öffentlichkeit gegenüber die Funktion eines "diachronen Korrektivs". Ich werde diesen Vorschlag an zwei Beispielen zu veranschaulichen suchen.

\section{Beispiel 1:}

\section{Katholizismus oder: die mediale Rezeption eines Klischees}

Das Jahr 1992 war nicht nur das Jahr der Bibel, es war auch das Jahr des Qumran-Bestsellers „Verschlußsache Jesus" der beiden amerikanischen Journalisten Michael Baigent und Richard Leigh.8 Die Thesen dieses Buches haben die öffentliche Diskussion erheblich bestimmt: den Inhalt des Buches könnte man sehr schnell vergessen, wenn nicht eine enorme Rezeption bis hinein in die Intensivsegmente des Katholizismus zu verzeichnen gewesen wäre. ${ }^{9} \mathrm{Im}$ letzten steckt hinter diesem Buch eine fiktionale Handlung: John Allegro, der dem internationalen ForschungsTeam der Herausgeber der Qumranfunde angehörte, nahm darin eine Minderheitenposition ein; er edierte nicht nur seine Texte miserabel, startete nicht nur eine Ausgrabungskampagne auf eigene Faust, sondern brachte 1966 ein Bühnenstück mit dem Titel „Die anfeuernden Orakel” heraus. In diesem Stück geht es um folgende Handlung: eine Universitätsassistentin löst unter reichlich Dampf zusammengeklebte Teile einer Qumranrolle auf. Der herbeigerufene Professor überfliegt die nun lesbaren Texte und konstatiert emphatisch: "Das wird die katholische Kirche erschüttern!" Professor Lanson alias John Allegro hatte in der aramäischen Rolle entziffert, daß Jesus Petrus nicht "kaiphas" (Fels), sondern lediglich "gajpha" (Untersucher) genannt hatte. Mit diesem Fund sei das Papsttum mehr oder weniger erledigt. Die katholische Assistentin ist wegen ihrer Mit-täterschaft erschüttert und beichtet diesen Vorfall einem Priester. Dieser unterrichtet sofort katholische Konspiration par excellance - die vatikanischen Behörden, welche durch Mittelsmänner den Professor von nun an beschatten. Sie setzen ihn unter Druck, er solle doch wieder "Fels“ lesen. Der Professor freilich bleibt standhaft. Er will die Öffentlichkeit von der Richtigkeit seiner Position überzeugen. Als er bei einer einberufenen Pressekonferenz das Geheimnis lüften will, ist allerdings der Safe, in dem die Qumran-Rolle lagerte, leer. "So unterdrückt man die Worte Jesu“, mit diesem Ausruf des Professors endet das Stück von John Allegro.

Diese Handlung liest sich wie das Drehbuch zur "Verschlußsache Jesus." Eine fiktionale Handlung mit ganz bestimmten antikatholischen Wertungsmustern wird zum Faktum: aus einem Bühnenstück mit

8 M. Baigent/R. Leigh, Verschlußsache Jesus. Die Qumranrollen und die Wahrheit über das frühe Christentum, München 1991.

9 Vgl. dazu E. Garhammer, Von der Fiktionalität zur Faktizität. Analyse momentaner Kirchenkritik am Beispiel des Qumran-Themas, in: ThGl 84 (1994) 37-46. 
erfundener Handlung ist ein Sachbuch geworden, aus Fiktionalität Faktizität. Alle Handlungsmomente des Sachbuches sind im Drama schon enthalten: sensationelle Entdeckungen in den Qumran-Rollen, die die Kirche bedrohen. Diese Funde freilich werden durch die VatikanMafia unterdrückt. Nun aber wird die Wahrheit - im Gegensatz zur Bühnenhandlung - in Form der mobilisierten öffentlichen Meinung siegen - trotz der Machenschaften des Vatikans. Wer das Buch von Baigent und Leigh liest, erfährt die Wahrheit über das Christentum - so auch der Text der Verlagswerbung: „Die Wahrheit über das frühe Christentum - vom Vatikan unterdrückt. Diese Dokumente enthüllen, daß die Anfänge des Christentums falsch überliefert worden sind.“ 10

Nun hat der Erfolg dieses Buches freilich noch andere, komplexere Hintergründe, die hier nicht näher analysiert werden sollen. Inhaltlich bietet es ein Bild des Katholizismus mit folgenden Behauptungen:

1. Katholischer Glaube und Wissenschaftlichkeit schließen sich aus:

2. Katholischer Glaube und abweichende Meinungen sind nicht kompatibel. Andersdenkende werden stets isoliert und diskriminiert.

3. Der katholische Glaube erlaubt keine freie Meinung, sondern ist ein Diktat des Vatikans. Vatikan und Glaubenskongregation sind ausgeklügelte Kontrollsysteme.

4. Der katholische Glaube verlangt im letzten ein "sacrificium intellectus."

Als praktischer Theologe steht man - auch wenn fachwissenschaftlich die Erkenntnisse des Buches längst falsifiziert sind - ziemlich ratlos vor seinem Erfolg, zumal Richtigstellungen von theologischer Seite sehr schnell als kirchliche Apologetik abgetan werden.

Pastoraltheologie als Vermittlerin von kulturellem und kommunikativem Gedächtnis hat die Aufgabe, die Geschichte zu befragen, ob es ein Vorurteilsmuster gibt, das sich hier repetiert. Ich möchte paradigmatisch zwei Vorgänge schildern, die ein ähnliches Bild des Katholizismus transportieren - allerdings in einem wesentlich eingeschränkteren Öffentlichkeitstypus, nämlich dem philosophischen und literarischen Milieu.

\section{Stolbergs Konversion zum Katholizismus im Jahre 1800}

Als Friedrich Leopold Graf zu Stolberg am Pfingstsonntag des Jahres 1800 in der Hauskapelle der Fürstin Gallitzin in Münster den Übertritt in die katholische Kirche vollzog, sorgte er nicht nur in seinem

10 Vgl. dazu E. Garhammer, Qumran und die Vermarktungsmechanismen der Medien. Oder: Warum eine Medienschelte nur die halbe Wahrheit ist!, in: ThGl 84 (1994) 151-158. 
Freundeskreis, sondern in der gesamten kulturellen Öffentlichkeit für Aufregung.11 War dieser Schritt doch etwas anderes als die bisher bekannten romantischen Konversionen. Wer aus beruflichen Gründen oder aus künstlerischen Motiven die Konfession wechselte, war der Toleranz der Aufklärer sicher. Zudem waren bis dato die Konversionen eher innerhalb von katholischen Majoritäten passiert. Stolberg bekleidete das Amt des Präsidenten des lutherischen Konsistoriums in Eutin und war leitender Beamter des Fürstbischofs von Lübeck. Für seinen Entschluß waren weder berufliche noch künstlerische Motive ausschlaggebend, sondern die Überzeugung, daß die katholische Kirche die allein wahre sei. Dies löste in der Öffentlichkeit einen für damals nicht unbeträchtlichen Skandal aus.

Äußerst bestürzt reagierte vor allem F. H. Jacobi, der seinem Freund bescheinigte, er könne es „unmöglich für eine redliche Überzeugung halten, wenn ein Evangelischer Papist “ werde. Seiner Meinung nach unterdrücke der römische Katholizismus die Gewissensfreiheit, mache Gott zu einem "lächerlichen Götzen" und erhebe den Klerus mit seinem Wirken über die göttliche Wahrheit selber. In einem Brief bescheinigt Jacobi den Katholiken "Lama-Dienst” und „Kothfressen." Er erläutert diesen Brief an einen Freund: Stolberg habe sich für ein System entschieden, welches die Unterwerfung der eigenen Vernunft unter die der Kirche fordere. Gegen solch ein System könne man nicht tolerant sein; eine so geartete Toleranz wäre "Gleichgültigkeit gegen Vernunft und wahre Religion" und würde der katholischen „inhumanen Verirrung des Geistes und des Herzens“ den Weg bereiten. Diese "Barbarey und Thyranney" müßte mit allen Mitteln bekämpft werden.12

Johann Heinrich Voß griff ebenfalls zur Feder und verfaßte ein Gedicht mit dem Titel „Warnung“. Eine Strophe darin lautet:

„Pfaffenknecht? Ab schwörest du Licht und Wahrheit?

Am Altarschmaus dann des gebackenen Gottes

Schnaubst du dem, was Menschen vom Thier erhebet, Haß und Verfolgung?" 13

Katholischsein heißt also für Voß: der "Vernunft abschwören". Herder urteilte zurückhaltender; er schrieb an die Ehefrau Luise Stolberg:

"Ohne Zweifel ists die Reise nach Italien, die den Keim des Uebels tief gelegt hatte. Der Graf ging mit zu voller gedrängter Seele in

11 Vgl. dazu D. W. Schumann, Aufnahme und Wirkung von Friedrich Leopold Stolbergs Übertritt zur katholischen Kirche, in: Euphorion 50 (1956) 271-306. Zum Kreis von Münster vgl. S. Sudhof, Von der Aufklärung zur Romantik. Die Geschichte des „Kreises von Münster", Berlin 1973.

12 Zit.n. D. W. Schumann, Aufnahme und Wirkung (Anm. 11), 286.

13 Ebd., 287. 
dieses verführerische Land; Kunst und Alterthum waren ihm zu ferne, zu todte Gegenstände, als daß sie ihn füllen, erwärmen konnten, und so übermannte ihn die so imposante Gegenwart des Cultus... Der Tod seines Kindes, andere Dinge ohne Zweifel, die ich nicht weiß, gruben diesen Eindruck tiefer ein... Finde er Ruhe unter einer Bürde, die so viele, viele brave Katholische als das ansehen was sie ist, ein Bündel von Gebräuchen und Mißbräuchen, das Jahrhunderte, dunkle Jahrhunderte zusammengebunden haben." 14

Bei aller Irenik, die in Herders Worten durchschimmert, wird doch auch deutlich, daß er die Konversion Stolbergs nur als Folge eines zerrissenen Gemüts deuten kann. Die Anstrengung des denkerischen, aber dadurch die Kräfte des Menschen fordernden Protestantismus sei ihm zuviel gewesen. Die Ruhe, die Stolberg nun gefunden habe, sei ihm zu gönnen, aber sie trage doch Züge einer Resignation.

In diesen Deutungen, die noch um etliche Stimmen vermehrt werden könnten, zeigen sich polemisch und irenisch ganz bestimmte (Vor-) Urteile über den Katholizismus:

1. der Katholizismus unterdrückt die Gewissensfreiheit

2. der Katholizismus stellt den Gehorsam über die Wahrheit

3. der Katholizismus macht den einzelnen zum Götzendiener des Papsttums

4. der Katholizismus macht aus einem vernünftigen Menschen einen Pfaffenknecht

5. der Katholizismus verschafft zwar Beruhigung des Geistes, aber nur um den Preis der Unterwerfung.

\section{Döblins Konversion zum Katholizismus im Jahre 1941}

Was sich $1800 \mathrm{im}$ theologisch-philosophischen Milieu abspielte, ereignete sich 1943 im literarischen: Am 14. August 1943 versammelt sich in einem Kino in Santa Monica bei Los Angeles die in diesem Landstrich ansässige künstlerische Prominenz des deutschen Exils. Die Brüder Heinrich und Thomas Mann sind ebenso anwesend wie Bert Brecht, Helene Weigel, Hanns Eisler, Arnold Schönberg, Fritz Kortner und viele andere. Man begeht den 65. Geburtstag von Alfred Döblin. Döblin war nach einer abenteuerlichen Flucht durch den Süden Frankreichs nach Nordamerika gekommen und wurde von seinen Kollegen dort aufgenommen. Nur eines hatte der Jude Döblin, der Autor des berühmten Romans "Berlin Alexanderplatz", seinen Freunden bis dato verschwiegen: auf der Flucht vor den Nazis, in einer Situation schwerer innerer

14 S. Sudhof, Herder und der „Kreis von Münster". Ein Beitrag zur Beurteilung von F. L. Stolbergs Konversion, in: Literaturwissenschaftliches Jahrbuch N. F. 1 (1960) 133-141. Der Brief Herders vom 29.09.1800 ist abgedruckt ebd., 141-143, hier 141f. 
Kämpfe, war es in der Kathedrale von Mende zu einem religiösen Erlebnis, zu einer tiefen Begegnung mit dem Gekreuzigten gekommen; Döblin schildert es in seinen autobiographischen Aufzeichnungen so:

„Und wieder vor einer Kirche! Man tritt ein, besichtigt sie. Mein Blick fällt auf das Kruzifix... meine Blicke wanderten zu dem, der dort schrecklich hing, fragten, kehrten leer wieder. Ich erinnere mich. Jetzt ist eine Vertraulichkeit da zwischen dem Kruzifix und mir. Es besteht ein Geheimnis zwischen uns. Der am Kreuz hängt, spricht nicht zu mir, aber ich spreche $z u$ ihm. Ich frage nicht. Ich fühle: Oh du! Ich bin auf der Flucht, und da ist man gehetzt und es gibt Grauen. Aber was ist das, verglichen mit dem Grauen, durch das du hast gehen müssen auf unserer Erde, unter uns Menschen. “15

Döblin beschreibt weiter, daß er diese Begegnung nicht mehr vergessen konnte. Nach seiner Ankunft in Kalifornien begann er mit theologischen Studien und ließ sich von Jesuiten in die katholische Kirche aufnehmen. Am 30. November 1941 empfing er in einer katholischen Kirche in Hollywood die Taufe.

Eineinhalb Jahre später, also an dem berichteten 14. August 1943, sieht Döblin den Zeitpunkt seines 65. Geburtstags als günstigen Anlaß, seine Glaubensbekehrung öffentlich bekanntzugeben. Diese Sätze - die im Wortlaut nicht überliefert sind - verletzten den guten Geschmack der Versammelten und wurden als äußerst peinlich empfunden. Bei manchen war die Empörung so groß, daß sie unmittelbar an diesem Abend noch zur Feder griffen. So trug Bert Brecht noch am gleichen Abend in sein Arbeitsjournal folgende Sätze ein:

„helli (= Weigel) hat eine feier für Döblin organisiert, der 65 jahre alt wurde. heinrich mann hielt eine herrliche begrüßungsrede... und am schluß hielt döblin eine rede gegen moralischen relativismus und für feste maße religiöser art, womit er die irreligiösen gefühle der meisten feiernden verletzte. ein fatales gefühl ergriff die rationaleren zuhörer, etwas von dem verständnisvollen entsetzen über einen mitgefangenen, der den folterungen erlegen ist und nun aussagt. tatsächlich haben besonders harte schläge döblin niedergeworfen: der verlust zweier söhne in frankreich, die undruckbarkeit eines 2400 - seiten - epos, angina pectoris (die große bekehrerin) und das leben mit einer ungewöhnlich dummen und spießigen frau.

als döblin anfing zu beschreiben, wie mit vielen anderen schreibern auch er mitschuldig wurde an dem aufstieg der nazis... und die frage entschlossen aufwarf, warum denn, glaubte ich für minuten kindlich, er werde jetzt fortfahren: „weil ich die verbrechen der

15 A. Döblin, Autobiographische Schriften und Aufzeichnungen, Olten 1977, 284. 
herrschenden vertuscht, die bedrückten entmutigt, die hungernden mit gesängen abgespeist habe“ usw. aber er fuhr nur verstockt, unbußfertig, ohne reue fort: „weil ich gott nicht suchte.“16

Der biblische Begriff der Verstockheit wird hier von Brecht auf den Glaubenden bezogen. Wenig später verarbeitete derselbe Brecht diesen Vorgang noch einmal in einem Gedicht, überschrieben:

"Peinlicher Vorfall“:

Als einer meiner höchsten Götter seinen 10.000 Geburtstag beging Kam ich mit meinen Freunden und meinen Schülern, ihn zu feiern

Und sie tanzeten und sangen vor ihm und sagten Geschriebenes auf.

Die Stimmung war gerührt. Das Fest nahte seinem Ende.

Da betrat der Gefeierte Gott die Plattform, die den Künstlern gehört und erklärte mit lauter Stimme

Vor meinen schweißgebadeten Freunden und Schülern

$\mathrm{Da}$ er soeben eine Erleuchtung erlitten habe und nunmehr

Religiös geworden sei und mit unziemlicher Hast

Setzte er sich herausfordernd einen mottenzerfressenen Pfaffenhut auf

Ging unzüchtig auf die Knie nieder und stimmte

Schamlos ein freches Kirchenlied an, so die irreligiösen Gefühle

Seiner Zuhörer verletzend, unter denen

Jugendliche waren.

Seit drei Tagen

habe ich nicht gewagt, meinen Freunden und Schülern

unter die Augen zu treten, so

Schämte ich mich. ${ }^{17}$

Brecht vulgarisiert und popularisiert die Ansichten der Religionskritik des 19. Jahrhunderts mit den Mitteln der Literatur:

- die religiösen und biblischen Sprachspuren in diesem Gedicht dienen eher der Parodie, der Satire und der ironischen Kommentierung des Geschehens. "Sie tanzeten und sangen" läßt den Tanz um das Goldene Kalb assoziieren, die laute Stimme den Kreuzesschrei Jesu, der hier allerdings in ein freches Kirchenlied mündet, nicht in einen Klagepsalm.

- Brecht arbeitet mit dem Mittel der Verkehrung. Religiöses Vokabular

16 B. Brecht, Arbeitsjournal, Bd. 2: 1942-1955, hg.v. W. Hecht, Frankfurt/M. $1973,605$.

17 B. Brecht, Gesammelte Werke (Suhrkamp) 10, 86f. Vgl. dazu E. Garhammer, "Sie werden lachen - die Bibel." Bibel und moderne Literatur, in: $H$. Frankemölle (Hg.), Die Bibel. Das bekannte Buch - das fremde Buch, Paderborn u.a. 1994, 111-128. 
wird von ihm für die eigene irreligiöse Position in Dienst genommen. Das öffentliche religiöse Bekenntnis verletzt die Gefühle der Zuhörer, für die der Jugendschutzparagraph bemüht wird. Brecht reklamiert für seine Irreligiösität das, was vorher Privileg des Religiösen war: Scham und Ehrfurcht.

- Während der Irreligiöse schamhaft schweigt, biedert sich der Gläubige schamlos und unzüchtig an. Gegen eine religiöse Pornographie steht eine irreligiöse Keuschheit.

- Im Arbeitsjournal bringt Brecht seine Einstellung zur Religiösität noch klarer zum Ausdruck: er deutet Religiosität psycho-pathologisch. Wer religiös ist, wird mit seinen Lebenskrisen nicht fertig.

- Ein weiterer Vorwurf Brechts an Döblin lautet: er hat die unter Künstlern geltenden Spielregeln verletzt, er hat die Bühne zu einer Kanzel gemacht, das Theater zu einer Kirche umfunktioniert. Er hat den Freiraum der Kunst dazu mißbraucht, religiöse Propaganda zu betreiben. So ist aus dem gefeierten Gott ein Pfaffenknecht geworden.

Dabei kann man leicht nachweisen, daß die Frage nach Gott eine durchgehende Spur in Döblins Leben darstellt. Schon 1902 wird durch die Nietzsche-Lektüre die modische Haltung des distanzierten Intellektuellen, der sich religiöser Aussagen enthält, in Frage gestellt:

"Ich erinnere mich, wie ich im Zimmer sitze und nach der Lektüre der 'Genealogie der Moral' das Buch schließe, beiseitelege und mit einem Heft bedecke, buchstäblich zitternd, fröstelnd, und wie ich aufstehe, außer mir, im Zimmer auf und abgehe und am Ofen stehe. Ich wußte nicht, was mir geschah, was man mir hier antat. Kannte ich Gott, trotz alledem? Gott, gegen den es hier ging? Wußte ich von ihm? Ahnte und ersehnte ich ihn? Ich weiß nicht. Aber ich sah, daß es hier schrecklich ernst wurde, daß es um Gott ging, und daß ich daran beteiligt war." 18

Das damit aufgebrochene Gottesproblem ließ Döblin nicht mehr los, es wurde sogar noch gesteigert durch das Studium von Freud und Marx; die Auseinandersetzungen mit den drei Meistern des Zweifels und des Verdachts, wie P. Ricoeur Nietzsche, Freud und Marx genannt hat, führte Döblin auf die Spur des Betens. Er bezeichnet in seinen autobiographischen Schriften seine Werke in gewisser Weise alle als

18 Vgl. dazu H. Kiesel, Literarische Trauerarbeit. Das Exil- und Spätwerk Alfred Döblins, Tübingen 1986, 146 sowie ders., Döblins Konversion als Politikum, in: F. Gaede/P. O'Neill/U. Scheck (Hg.), Hinter dem schwarzen Vorhang. Die Katastrophe und die epische Tradition (FS Riley), Tübingen 1994, 193-208. 
Gebete. Als er 1904 zur medizinischen Promotion nach Freiburg reiste, besuchte er das Münster: "Ich bin gestern zum Hochamt im Münster gewesen, nachmittags bin ich noch einmal allein in das dunkle leere Gewölbe zurückgegangen. Das Beste, was wir können, ist beten. Im Grunde beten wir ja immerfort. Nichts ist mir widriger, als der aufgeklärte Liberalismus, der über die Religionen lacht und sie für Massenfraß hält. Das Beste, was wir können, ist beten," so Döblin in einem Brief vom 4. November 1904 an die Schriftstellerin Else Laske-Schüler. ${ }^{19}$

Auf diesem Hintergrund wird deutlich, daß das Bekehrungserlebnis von Döblin sich nicht auf eine Augenblicksentscheidung zusammenziehen läßt. Der Meditation des Kreuzes in der Kathedrale von Mende waren längst andere Erlebnisse vorangegangen, etwa die Beschreibung des Kruzifixes von Veit Stoß in der Krakauer Marienkirche. Ein Jahr nach dem Kreuzeserlebnis in Krakau schrieb Döblin 1926: „Es kommt mir vor, als ob das heutige Christentum... das ungeheure fabelhafte Faktum des Jesus von Nazareth nicht genügend ausmünzt, und daß das Ungeheure und Beseligende dieser Erscheinung nicht lebendig genug gelebt wird. Es müßte eigentlich ein Strom von Gefühl und Tatkraft aus dieser Gestalt hertosen. "20

Döblins Religiösität kann nicht - wie es Brecht denunziatorisch getan hat - psychopathologisch erklärt werden: das hieße, die konsequente Entfaltung der religiösen Dimension in Döblins Werk außer acht lassen. Die strikte Entgegensetzung von Glaube und politischem Engagement durch Brecht ist ebenfalls verkürzend und blind gegenüber der Möglichkeit einer praktisch und politisch wirksamen Religiösität. Die Literaturgeschichtsschreibung ist durch die Abwertung des Döblinschen Spätwerks im Grunde genommen bis heute dem Diktum Brechts gefolgt.

In den Vorgängen um die Konversion Döblins und den damit zusammenhängenden Deutungen in der literarischen Öffentlichkeit kommt ein Urteil über den Katholizismus zum Vorschein, das wir zwischenzeitlich schon kennen:

1. wer katholisch wird, wird ein Pfaffenknecht,

2. wer katholisch wird, hält die Probleme des Lebens intellektuell nicht aus,

3. wer katholisch wird, setzt gegen die "religiöse Keuschheit" der liberalen Distanz die "religiöse Pornographie" des öffentlichen Bekenntnisses.

19 H. Kiesel, Literarische Trauerarbeit (Anm. 18), 154.

20 Ebd., 185. 
Mit diesen Beispielen sollte gezeigt werden, wie ein bestimmtes Bild des Katholizismus, das im philosophischen und literarischen Milieu längst gang und gäbe war, durch den Einfluß der Medien in ganz neue Schichten transportiert wird.

Der Pastoraltheologe nimmt wahr, wie sich solche Deutungsmuster des Katholizismus, von denen ich einige aufgezählt habe, heute durch die Medien bis in den Bereich der Engagierten in den Pfarrgemeinden durchsetzen: es hat sich ein Kommunikationsstil entwickelt, den man so beschreiben könnte: Enthüllung durch die Medien - Verteidigung durch kirchliche Verlautbarungen oder: revelatorische Dimension der Medien und velatorische Anstrengung der Kirchen.

Dabei ist klar: den Einfluß der Medien kann man nicht durch Beklagen verändern, die Auseinandersetzung mit ihnen muß vielmehr kommunikativ geführt werden. Es wurden allerdings innerkirchlich, was Kommunikation angeht, in der Vergangenheit hauptsächlich zwei Modelle entwickelt: das eisige Schweigen, die kommunikative Verweigerung, oder das Kulturkampfmodell, das den Kritikern sofort die Vernichtung der Kirche unterstellt, der man nur durch eine "acies formata" begegnen kann, nämlich durch Sammlung und Verpflichtung zur einheitlichen Sprachregelung.

Ein drittes Modell, das ich "kommunikative Diakonie“, nennen möchte, steht erst am Anfang. Pastoraltheologie müßte diese kommunikative Diakonie innerkirchlich realisieren helfen. Bei diesem Vorschlag einer "kommunikativen Diakonie" ist es nicht damit getan, hehre Forderungen aufzustellen, sondern - wie bei der Diakonie üblich - Schritte anzuzielen, die ins eigene Fleisch schneiden.

Solche Schritte könnten sein: einige Mitglieder der Deutschen Bischofskonferenz geloben ein "Bußschweigen“ in den Medien. Gerade die Kollision von bestimmten Bischöfen und den Medien - man kann dies eine symmetrische Verteufelungseskalation nennen - stabilisiert die gegenseitigen Feindbildmuster.

Ein weiterer Schritt könnte darin bestehen, daß sich einige Mitglieder der Pastoraltheologenkonferenz auf ein "Bußschweigen“ bei päpstlichen Erlassen verpflichten. Dadurch könnte die hier laufende Transaktion - autoritäres Vater-Ich und rebellierendes Kinder-Ich aufgelöst werden. Statt dessen sollten die betroffenen Theologen in jedem Jahr von einer Ortsgemeinde zu einer Fastenpredigtreihe eingeladen werden und die Fastenzeit dort mitbegehen.

Ein weiterer Schritt könnte heißen: die bischöflichen Pressestellen laden nicht nur jedes Jahr die im Bistum ansässigen JournalistInnen zu 
einem Stehempfang ein, sondern zur Teilnahme am bischöflichen und gemeindlichen Alltag (Firmungsreise, Visitation, caritative Projekte u.ä.).

Nur wenn es gelingt, daß beide Seiten - Kirche und Medien - ihre Wahrnehmungsmuster überprüfen, ist neue Kommunikation möglich. Dies setzt aber "Sündenbekenntnis" und Umkehr auf beiden Seiten voraus. Der Dogmatiker Siegfried Wiedenhofer fordert in ähnlicher Weise eine neue Form von Apologetik: nicht mehr nur rationale, im Sinn von Vorwurf und Verteidigung betriebene, sondern vor allem spirituelle Apologetik, in Form von eigener Umkehr und Vergebungsbitte.21 Hinter meinem Vorschlag verbirgt sich nicht die Absicht, kirchliche Kommunikation der medialen anzupassen, genau das Gegenteil wird angezielt. In diesem Zusammenhang will ich darauf hinweisen, $\mathrm{da} B$ es ein dringendes Desiderat darstellt, einen theologischen Öffentlichkeitsbegriff zu erarbeiten. Wichtige Hinweise dafür finden sich bei Erik Peterson, der feststellte: "Nur die Herren dieser Welt kennen ein arcanum: nämlich Machterhalt und Geldbesitz." 22 Christen, die auf die Parusie warten, also auf eine universale Öffentlichkeit, steht solches Geheimhalten schlecht an. Der Freiburger Weihbischof Paul Wehrle hat in dieser Richtung ebenfalls richtungsweisende Thesen entwickelt. Er stellt fest: "Die Zukunft der Kirche wird wesentlich mit einer Aufarbeitung und Verbesserung der innerkirchlichen Kommunikation zusammenhängen. ${ }^{23}$

21 S. Wiedenhofer, Apologie der Kirche - Idealisierung der Kirchengeschichte?, in: H. R. Seeliger (Hg.), Krimininalisierung des Christentums? Karlheinz Deschners Kirchengeschichte auf dem Prüfstand, Freiburg 1993, 97-112. Das päpstliche Schreiben "Tertio millenio adveniente" zur Vorbereitung auf das Jubeljahr 2000 schlägt solche neuen Töne bereits an: „Zu Recht nimmt sich daher die Kirche, während sich das zweite christliche Jahrtausend seinem Ende zuneigt, mit stärkerer Bewußtheit der Schuld ihrer Söhne und Töchter an, eingedenk all jener Vorkommnisse im Laufe der Geschichte, wo diese sich vom Geist Christi und seines Evangeliums dadurch entfernt haben, daß sie der Welt statt eines an den Werten des Glaubens inspirierten Lebenszeugnisses den Anblick von Denk- und Handlungsweisen boten, die geradezu Formen eines Gegenzeugnisses und Skandals darstellten." (in: Verlautbarungen des Apostolischen Stuhls Nr. 118, hg.v. Sekretariat der Deutschen Bischofskonferenz, 29).

22 Vgl. dazu B. Nichtweiß, Offenbarung und Öffentlichkeit. Herausforderung der Theologie Erik Petersons, in: Jahres- und Tagungsbericht der GörresGesellschaft 1993, 77-106 sowie L. Hölscher, Öffentlichkeit und Geheimnis. Eine begriffsgeschichtliche Untersuchung zur Entstehung der Öffentlichkeit in der frühen Neuzeit, Stuttgart 1979.

23 Vgl. dazu P. Wehrle, Zukunft der Kiche - Kirche der Zukunft, in: LS 45 (1994) 6-14, hier 11. Der Präsident der DFG, Prof. Dr. Wolfgang Frühwald, forderte in seinem Festvortrag anläßlich des 500jährigen Jubiläums des Herzoglichen Georgianums in München von Kirche und Theologie bei dem erforderlichen Dialog mit heutiger Welt eine Selbstentäußerung, "welche die zeitweilige Suspendierung uralter Denktraditionen voraussetzt, damit über- 


\section{Beispiel 2: \\ Verdrängung des Todes oder: die theologische Rezeption eines Klischees}

In einem kürzeren zweiten Beispiel soll gezeigt werden, wie die Medien sich durchaus mit den "Zeichen der Zeit" auseinandersetzen. Dazu gehören mit Sicherheit Tod und Sterben in unserer Gesellschaft. Die Filmemacherin und Schriftstellerin Doris Dörrie hat in ihrem Film "Keiner liebt mich" die Auseinandersetzung mit Tod und Sterben in der heutigen Gesellschaft zu visualisieren versucht; sie nimmt dabei die "ars moriendi" der Moderne, nämlich das Sterben vorweg zu erleben, auf die Schippe. ${ }^{24}$ In diesem Film wird deutlich, daß die Thesen von der Verdrängung des Todes in der Moderne, die innertheologisch immer noch transportiert werden, in dieser Eindeutigkeit und Zuspitzung nicht mehr zutreffen.25 Es gibt eine ganz neue Todesdeutung, wie sie die Literatin Angelika Mechtel in ihrem Buch "Die Prinzipalin" ebenfalls vorgenommen hat: die Umdeutung vom Sensenmann in einen Liebhaber. In dem genannten Roman schildert A. Mechtel doppelperspektivisch das Leben der Theaterprinzipalin Caroline Neuber (1697-1760) sowie ihre Auseinandersetzung mit dem Tod; die Obsession des Theaters läßt die Neuberin selbst im Sterben nicht los: sie fordert den eintretenden Tod auf, sich umzuschminken und seine Rolle vom Sensenmann in die des Liebhabers umzulernen. "Ich könnte dir beibringen, deine Rolle anders zu spielen. Im Liebhaberfach, denke ich, wärst du am besten aufgehoben... Maske und Kostüm werden wir ändern... Ein junger Tod. Betörend schön. Möchtest du dich darauf einlassen, mein Freund?"26

Pastoraltheologie müßte hier deutlich machen, daß es solches Umdeuten von Tod und Sterben immer schon gegeben hat. Ein sprechendes Beispiel ist Lessings Schrift „Wie die Alten den Tod gebildet", in der er gegen die Dramatik der barocken Todesauffassung

haupt eine gemeinsame Gesprächsebene gefunden werden kann“ (zit.n.Manuskript, 11).

Ähnlich ders., Die Dekade des Gehirns. Von den Schwierigkeiten des Strukturdialogs, in: A. Schavan (Hg.), Dialog statt Dialogverweigerung. lmpulse für eine zukunftsfähige Kirche, Freiburg 1994, 139-149.

24 Vgl. ihre zugrunde gelegte Geschichte "Orfeo", in: D. Dörrie, Für immer und ewig. Eine Art Reigen, Zürich 1993, 50-84.

Zum Film vgl. A. Kilb, Vom großen und vom kleinen Tod, in: Die Zeit, Nr. 3, 1995, 45f sowie "Sag lächelnd Good bye“, in: Spiegel 6/1995, 114-121.

25 Vgl. A. Heller, Ambivalenzen des Sterbens - Einschätzungen zum gegenwärtigen Umgang mit Sterben und den Sterbenden, in: ders. (Hg.), Kultur des Sterbens. Bedingungen für das Lebensende gestalten, Freiburg 1994, 13-32.

26 A. Mechtel, Die Prinzipalin, Frankfurt/M. ?1994, 106. Vgl. zu diesem Motiv in Literatur und Malerei G. Kaiser, Der Tod und die schönen Frauen. Ein elementares Motiv der europäischen Kultur, Frankfurt/M.-New York 1995. 
die aufklärerische Schlafhypothese setzte, die er der antiken HypnosTheorie entnahm.

„Totsein hat nichts Schreckliches; und insofern Sterben nichts als der Schritt zum Totsein ist, kann auch das Sterben nichts Schreckliches haben." 27 Visualisiert wurde diese Auffassung Lessings in der aufklärerischen Sepulkralskulptur J.G. Schadows. ${ }^{28}$

Eine ähnliche Umdeutung hatte es schon im Mittelalter gegeben: die apokryphe Erzählung vom Marientod diente dazu, die Apostel am Sterbebett Mariens erscheinen zu lassen und somit der Gottesmutter die mittelalterlich normierte "ars moriendi“ zu ermöglichen. ${ }^{29}$ Nach dem Zeitalter der Pest, in dem sich die Angehörigen von den Sterbenden zurückzogen, sollte die Wichtigkeit der Sterbebegleitung vor Augen geführt werden. Daraus wird deutlich: menschliche Grunderfahrungen - wie lieben und sterben - haben neben ihrer Einmaligkeit immer auch einen Zeitindex: in ihnen zeigen sich sowohl unhintergehbare Konstanten als auch plastisch zu haltende Ausdrucksformen. Pastoraltheologie hat den Druck und die Nötigung der Norm genauso kritisch zu analysieren wie den Zwang der Mode aufzudecken.

Für die Pastoraltheologie in diesem Verständnis stellt sich also nicht nur die Aufgabe, die modischen Themen der Medien einzuordnen und geschichtlich zu depotenzieren; sie hat ebenfalls den Anspruch, die Themen der Theologie mit dem aktuellen Diskurs in kritischem Austausch zu halten. Pastoraltheologie schützt mit diesem Ansatz nicht nur die Theologie vor der Gefahr der permanenten Selbstzitation, sie konfrontiert auch das Kurzzeitgedächtnis der Medien mit dem Langzeitgedächtnis der Kirche. Pastoraltheologie als Vermittlerin von kulturellem und kommunikativem Gedächtnis: dieser hier entfaltete Vorschlag erfordert intra- und interdisziplinäre Zusammenarbeit. ${ }^{30}$

27 G. E. Lessing, Wie die Alten den Tod gebildet. Eine Untersuchung, in: G. E. Lessing, Gesammelte Werke, hg.v. W. Stammler, 2. Bd., München 1959, 963-1015, hier: 999.

28 Vgl. dazu C. Keisch, "... den Tod gebildet?" - Schadows Grabskulpturen, in: B. Maaz (Hg.), J. G. Schadow und die Kunst seiner Zeit, Köln 1994, 113-121. Für die momentane "Ästhetisierung des Todes" ist der Befund von G. Schulze zu beachten, der der Erlebnisgesellschaft bescheinigt, Kontrastphänomene entweder zu marginalisieren oder zu ästhetisieren. Vgl. dazu G. Schulze, Die Erlebnisgesellschaft. Kultursoziologie der Gegenwart, Frankfurt/M.-New York 1992, 67-71.

29 Vgl. dazu K. Schreiner, Der Tod Marias als Inbegriff christlichen Sterbens. Sterbekunst im Spiegel mittelalterlicher Legendenbildung: A. Borst $(\mathrm{Hg}$.$) ,$ Tod im Mittelalter, Konstanz 1993, 261-312. „Der Tod Marias, wie er im Lichte zeitgebundener Vorstellungen, Normen und Rituale beschrieben dargestellt wurde, gab kirchlichen Sterberitualen des Mittelalters eine heilsgeschichtliche Dimension." (271)

30 Für die intradisziplinäre Zusammenarbeit sind alle theologischen Fächer relevant. Momentan scheint mir vor allem die Kirchengeschichte als 
SUMMARY: Cultural and communicative memory - Reflections on the self-unterstanding of pastoral theology in media-society

Pastoral theology is critized by observers for being a modern disciplin more obliged to modern than to theological reflections. The author in this article tries to proof that pastoral theology could fulfil a special task in media-society: to mediate between the cultural and the communicative memory (according to Jan Assmann). Doing this, pastoral theology could save theology from redundance on one side and save media from the building of clichees. In this way, pastoral theology would be a synchrone corrective for theology and a diachrone one for media.

RÉSUMÉ: Mémoire culturelle et communicative. Réfexions sur la théologie pastorale comme quelque chose d'évident dans un société médiatisée

Les critiques reprochent à la théologie pastorale d'être une discipline des temps modernes devant donc s'en tenir au discours moderne plutôt qu'au discours théologique. L'auteur essaie de prouver que la théologie pastorale pourrait remplir une tâche très spécifique dans un société médiatisée: Elle pourrait servir d'intermédiaire entre la memoire culturelle (Jan Assmann) et la mémoire communicative. Elle pourrait ainsi préserver la théologie du danger de la redondance, mais aussi attirer l'attention des médias sur la formation de clichés. La théologie pastorale serait donc un moyen d'amélioration synchronique pour la théologie, et un moyen d'amélioration diachronique pour les médias.

RESUMEN: Memoria cultural y comunicativa. Reflexiones referentes a la autocomprensión de la teologia pastoral en una sociedad de medios de masas

A la teologia pastoral sus criticos le enrostran que es una disciplina moderna y que por ello está más comprometida con la modernidad que con la teología. El autor intenta demostrar que la teología pastoral en una sociedad de medios de masa puede cumplir una tarea muy especifica: puede ser la intermediaria entre la memoria cultural (Jan Assmann) y la comunicativa. Así puede proteger a la teología del peligro de la redundancia, pero también puede hacer notar a los medios la formación de estereotipos: des esta manera la teología pastoral sería para la teología un correctivo sincrónico y para los medios uno diacrónico.

Ansprechpartnerin wichtig, weil sich in der Öffentlichkeit der Medien so etwas wie ein Dekadenzmodell des Christentums breit macht. Für den interdisziplinären Austausch ist es wichtig, daß es zu keinen einseitigen "Tandem-Bildungen” kommt. Vgl. zu diesem Problemkreis: F. Schweitzer, Praktische Theologie, Kultur der Gegenwart und die Sozialwissenschaften interdisziplinäre Beziehungen und die Einheit der Disziplin, in: K. E. Nipkow/D. Rössler/F. Schweitzer (Hg.), Praktische Theologie und Kultur der Gegenwart. Ein internationaler Dialog, Gütersloh 1991, 170-184. 